\title{
Numerical modelling of tectonic plates subduction using X-FEM
}

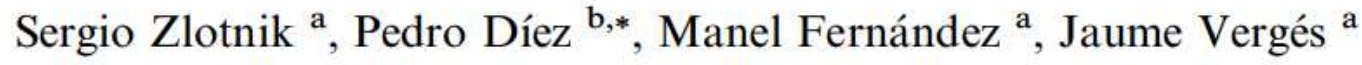 \\ ${ }^{a}$ Group of Dynamics of the Lithosphere (GDL), Institute of Earth Sciences "Jaume Almera", CSIC, Lluis Solé i Sabarís s/n, 08028 Barcelona, Spain \\ b Laboratori de Càlcul Numèric, Departament de Matemàtica Aplicada III, Universitat Politècnica de Catalunya, Campus Nord UPC, \\ 08034 Barcelona, Spain
}

\begin{abstract}
The numerical modelling of plate subduction requires solving a coupled thermo mechanical highly nonlinear transient problem. The mechanical description of the phenomenon results in a multiphase quasi static Stokes flow, where the inertia terms are neglected. The transient thermal problem is dominated by the advection term. Here, the representation and evolution of the different phases are described using level sets. The phase tracking is carried out transporting the level set along with the material, using a pure advective model. The gradient discontinuities induced by the viscosity jump across the interface are resolved numerically by enriching the solution using a partition of unity method in a eXtended Finite Element Method (X FEM) context. These numerical tools are used to simulate plate subduction with different parameters and to derive useful correlations between relevant geophysical factors.
\end{abstract}

Keywords: Tectonic plates; Subduction; Numerical modelling; EXtended Finite Element Method (X FEM)

\section{Introduction}

Plate tectonics is a framework in which most of the major Earth geological features can be explained. For instance, it explains satisfactory the global bathymetry, the existence of mid-ocean volcanic ridges, the position of continental mountain chains, the magnetic anomalies at the ocean bottom, the location of volcanic arcs and seismic activity, etc.

Despite the ability of assembling all these phenomena in a unified framework, major questions remain regarding the dynamics of the plates.

In subduction zones, oceanic plates bend and descend into the Earth's interior, generating most of the Earth's

\footnotetext{
Partially supported by Ministerio de Educación y Ciencia, Grants DPI2004 03000, CTM2005 08071 C03 03/MAR and CSD2006 00041.

* Corresponding author. Tel.: +34 934017240; fax: +34 934011825 .

E mail addresses: szlotnik@ija.csic.es (S. Zlotnik), pedro.diez@upc.es (P. Díez), mfernandez@ija.csic.es (M. Fernández), jverges@ija.csic.es (J. Vergés).

$U R L$ : www lacan upc.es (P. Díez).
}

volcanism and seismic activity. It is therefore important to understand subduction mechanisms and the history and current configuration of the plates in subduction zones. Numerical modelling of this phenomenon provides further insights into the mechanisms, as well as valuable information on the dependence of the results on material parameters and boundary conditions (velocities...).

The models used in simulations of these phenomena are essentially flow models with thermal coupling. The different plates and the underlying mantle are described as distinct phases with different material properties (viscosity, density, conductivity...). The most popular numerical technique used to track the phases in a finite difference or a finite element context, is the so-called marker and cell method [ [1 5 ]. This approach is limited by the lack of flexibility of the numerical discretization, and biased by the averaging function used to pass the marker's variables to the node's variables and vice versa.

In this work we use a level set technique to track the interface and to describe the phase motion. The phase tracking requires transporting the level with a pure 
advective model. Since the viscosity jump across the material interface produces a discontinuity in the derivatives of the velocity, we use the eXtended Finite Element Method (X-FEM) $[6,7]$ to interpolate the velocities, adding a functional enrichment via the partition of unity method. The XFEM approach has already been used to solve multiphase Stokes flows in other contexts (e.g. [8]).

The remainder of the paper is structured as follows. Section 2 is devoted to present the problem to be solved and to highlight the main assumptions and simplifying hypotheses. The proposed numerical methodologies are described in Section 3. A validation test is used in Section 4 to demonstrate the performance of the numerical solver of the mechanical problem and its desirable features. Finally, a number of simulations of subduction mechanisms for a set of different parameters is analyzed in Section 5.

\section{Problem statement}

The subduction process is a complex phenomenon in which some of the Earth's uppermost layers (lithospheric plates) penetrate into the Earth's interior (upper mantle). The mechanical behaviour of the system is modelled as a

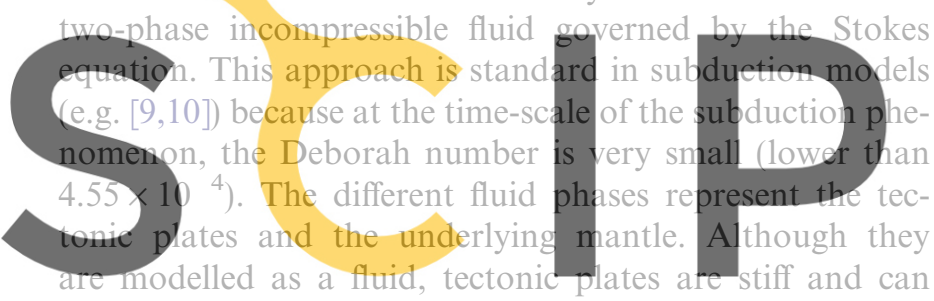
transmit stresses over long distances. This behaviour is

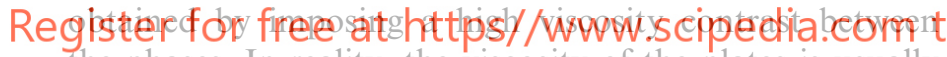
the phases. In reality, the viscosity of the plates is usually two to four orders of magnitude higher than the viscosity of the upper mantle. As previously mentioned, incompressibility for both phases is assumed.

Since the physical properties of rocks depend on temperature and pressure, subduction processes are highly influenced by their thermal state. In the domain under consideration, both temperature and pressure show drastic variations. For instance, in the upper $700 \mathrm{~km}$, temperature and pressure increase with depth, respectively, from $273 \mathrm{~K}$ and $0 \mathrm{GPa}$ at surface, to approximately $2000 \mathrm{~K}$, and $24 \mathrm{GPa}$ at the bottom. These extreme variations in temperature and pressure result in very different mechanical properties of the materials. For instance, the density of two chemically identical rocks, one near the surface and another at $700 \mathrm{~km}$ depth, may vary from $3200 \mathrm{~kg} / \mathrm{m}^{3}$ to $4900 \mathrm{~kg} / \mathrm{m}^{3}$. The rheological and thermal behaviour of the rocks are also imbricated. Consequently, in order to realistically simulate subduction phenomena, the mechanical and thermal models have to be coupled.

\subsection{Mechanical model}

As already mentioned, the mechanical part of the subduction system is modelled as an incompressible fluid gov- erned by the Stokes equation. Due to geophysical timescales and the viscosity orders of magnitude involved in this problem, dimensional analysis results in an extremely large Prandtl number (Prandtl number $10^{24}$ ). Therefore the inertia term is neglected and Stokes equation is considered in its quasi-static version:

$\nabla \cdot\left(\eta \nabla^{\mathrm{s}} \mathbf{u}\right)+\nabla p=\rho \mathbf{g}$,

$\nabla \cdot \mathbf{u}=0$

where $\mathbf{u}$ is the velocity, $\eta$ the viscosity, $p$ the pressure, $\rho$ the density, and $\mathbf{g}$ the gravitational acceleration vector. The operator $\nabla^{\mathrm{s}}$ is defined as $1 / 2\left(\nabla^{\mathrm{T}}+\nabla\right)$.

The nonlinear behaviour is introduced by the dependence of $\eta$ on the solution $\mathbf{u}$ and $p$. Moreover, $\eta$ is also a function of the temperature, and consequently the mechanical and thermal problem are coupled. Material properties are discussed in Section 2.3.

The transient equation (1) is said to be pseudo-static because it does not contain any explicit time dependence. The transient character of the solution is due to the motion of the phases and temperature field evolution.

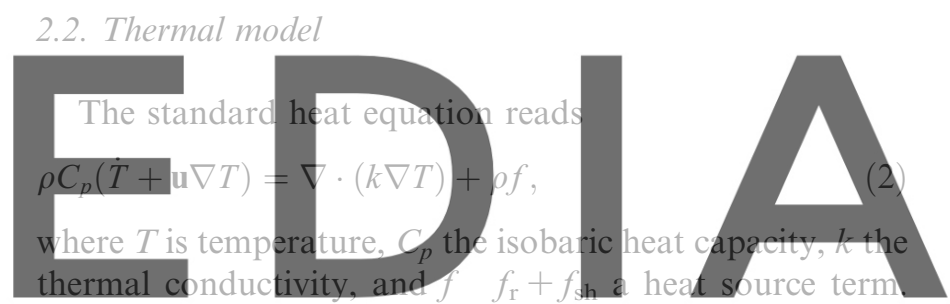

The later has two different components: the constant term

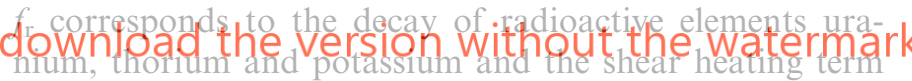
$f_{\mathrm{sh}}$ is associated with the mechanical heat dissipation. It is computed from the solution of the mechanical problem (1) as $f_{\text {sh }}=\sigma_{i j} \dot{\varepsilon}_{i j}$, where $\sigma$ is the deviatoric stress tensor $\sigma=2 \eta \dot{\varepsilon}$

and $\dot{\varepsilon}$ is the strain rate tensor

$\dot{\varepsilon}=1 / 2\left(\nabla \mathbf{u}+(\nabla \mathbf{u})^{\mathrm{T}}\right)$.

The dimensional analysis of Eq. (2) reveals that the convective term is three orders of magnitude larger than the diffusive term (associated with $k$ ). The thermal problem to be solved is therefore convection dominated.

\subsection{Material parameters}

The rheological and thermal properties of rocks are functions of temperature, pressure, and in some cases, strain rate. As a consequence of these dependencies, the mechanical and the thermal problem are two-way coupled.

\subsubsection{Density}

The proper assessment of the density $\rho$ is essential to the accurate simulation of subduction. In fact, the driving force is in this case generated by the contrast between the 
density of the cold subducting plate and the hot upper mantle. The resulting pulling force on the oceanic plate is of major importance in the system. The density is represented as a function of temperature and pressure by the following expression [11]:

$\rho=\rho_{0}\left[1-\alpha\left(T-T_{0}\right)\right]\left[1+\beta\left(p-p_{0}\right)\right]$,

where $\alpha$ and $\beta$ are, respectively, the thermal expansion and compressibility coefficients, and $T_{0}$ and $p_{0}$ are reference values at surface.

A mineral phase diagram indicates which are the stable mineral phases at any given temperature and pressure. The equilibrium Clapeyron line separates the stability fields of the three possible phases. That is, if two points (in the temperature pressure plane) are at different sides of the line given by the Clapeyron slope, then they correspond to different minerals. In this context, for our practical purposes, the phase diagram is divided into three regions corresponding to three minerals: olivine, spinel-structured olivine, and perovskite as shown in Fig. 1.

Two major mineralogical phase transitions occur, one at $410 \mathrm{~km}$ depth and other at $660 \mathrm{~km}$ depth (other deeper transitions run outside the domain under study). The den-

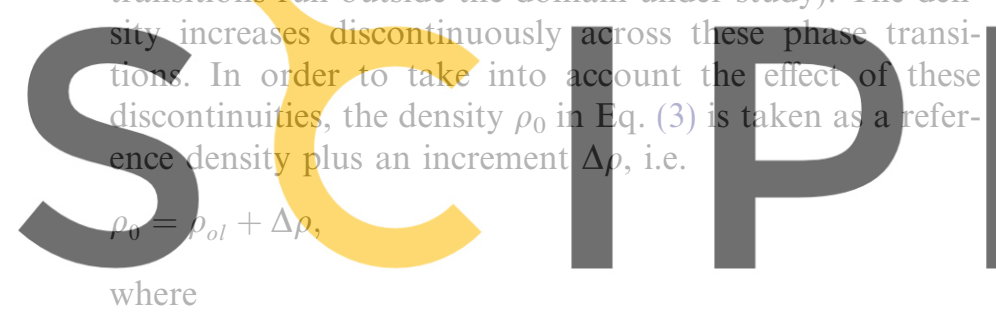

\section{Register fop free iatt httésih/wavavisiaipedia.com to $\Delta \rho= \begin{cases}\Delta \rho_{\mathrm{es}} & \text { if } T-p \text { is in the spinel region, } \\ \Delta \rho_{\mathrm{per}} & \text { if } T-p \text { is in the perovskite region. }\end{cases}$}

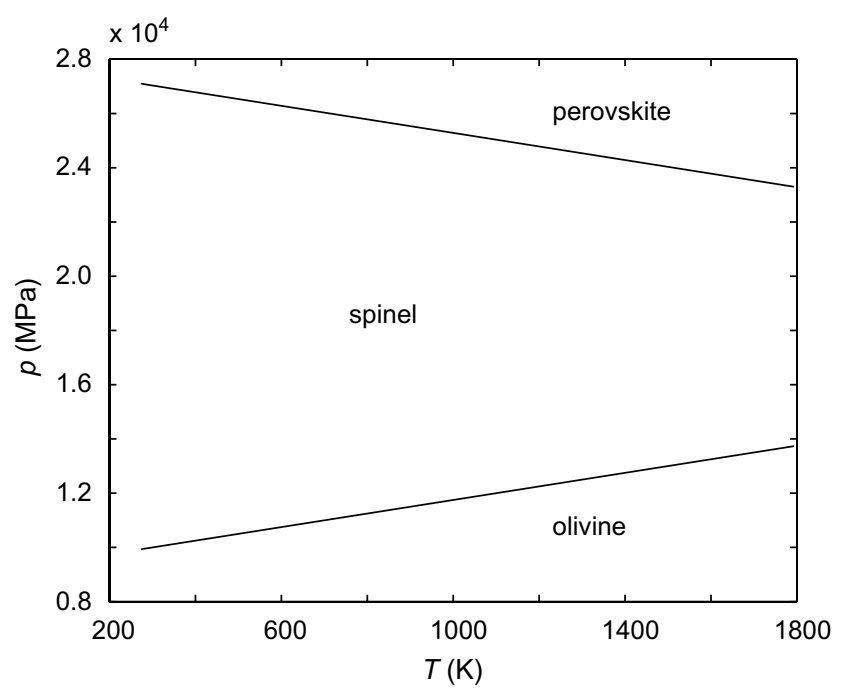

Fig. 1. Phase diagram indicating stable mineral phases in the tempera ture pressure plane. The phase diagram is divided into three regions corresponding to three distinct minerals: olivine, spinel and perovskite.

\subsubsection{Thermal conductivity}

The thermal conductivity of rocks also depends on temperature and pressure. At high temperatures $(T \geqslant 1500 \mathrm{~K})$ the electromagnetic radiation becomes important enough to be included as an extra heat transfer mechanism [12]. The following empirical expression, which includes both conductive and radiative effects, is used (see [13]):

$k=a+\left(\frac{b}{T+c}\right) \exp (d p)$

where $a \quad 0.73, b \quad 1293, c \quad 77$, and $d \quad 0.00004$, see [9]. Note that this expression is dimensional $\left(\mathrm{W} \mathrm{m}^{1} \mathrm{~K}^{1}\right)$ and therefore temperature and pressure have to be expressed in $\mathrm{K}$ and $\mathrm{MPa}$, respectively.

\subsubsection{Viscosity}

The mantle is assumed to behave mechanically as a nonNewtonian fluid with an average viscosity of $10^{21} \mathrm{~Pa}$. Note that, compared to the viscosity of honey at room temperature (19 $\mathrm{Pa} \mathrm{s}$ ), the mantle viscosity is 20 orders of magnitude larger.

The following power-law expression is used to describe how the viscosity depends on temperature, pressure, and

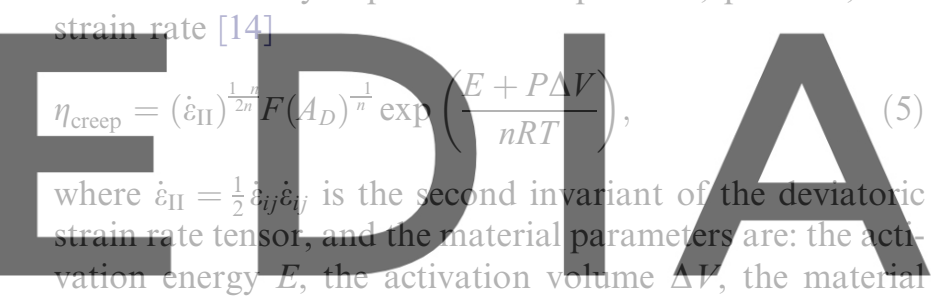

constant $A_{D}$, the stress exponent of the power-law $n$, and

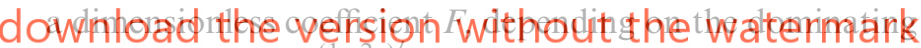

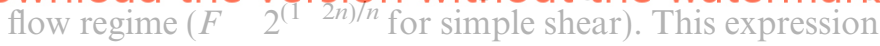
is truncated if the resulting viscosity exceeds a predefined threshold or is lower than a minimum value.

\subsection{Initial and boundary conditions}

The complete problem statement requires appropriate initial and boundary conditions for Eqs. (1) and (2).

The boundary conditions for the mechanical problem (1) are described in Fig. 2a. The velocity is imposed on the top of the domain. It is set to be zero on the left half, and it is given certain horizontal velocity on the right side. At the center point between these two domains, a downward velocity $\left(55^{\circ}\right.$ with the horizontal) is prescribed. A free slip condition is adopted at the bottom $\left(u_{z} \quad 0\right.$, zero shear forces). Along the laterals, labeled as $\Gamma_{s}$ on Fig. 2a, periodic boundary conditions are imposed. This allows material to flow trough the sides of the model in a confined domain. Finally, as a reference for the pressure field, a node on the surface is set to have null pressure.

The model domain is a 2D, $1000 \mathrm{~km}$ depth and $8000 \mathrm{~km}$ wide rectangle. The initial thermal state corresponds to that of a $100 \mathrm{~km}$ thick oceanic plate subducting beneath another plate with identical features (shown in Fig. 2b). The location of the material interface and the velocity field 


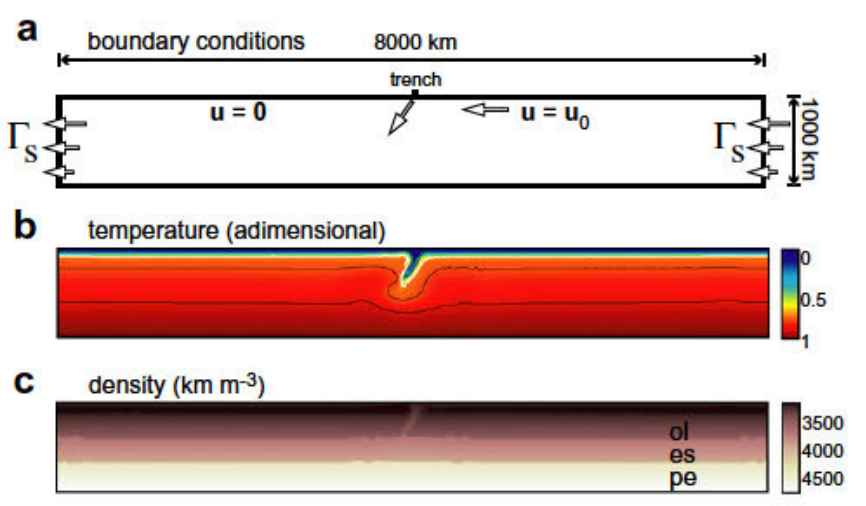

d viscosity $\left(\log _{10}(\mathrm{~Pa} \mathrm{sec})\right)$

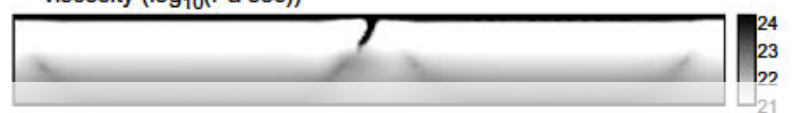

Fig. 2. Problem statement for the simulation of subduction: computa tional domain and boundary conditions (a); initial thermal state (b); initial density (c) (note that two discontinuities occur at 410 and $660 \mathrm{~km}$ depth) and initial viscosity (d).

are set accordingly to this configuration. The white line corresponds to the $1573 \mathrm{~K}$ isotherm which is the base of the tea nic plates. During simulation this sotherm chsely follows the interface defined by the are-the initial density and viscosi These ficlds are computed by a sintple she density has two sharr increments due to phase transitions at $410 \mathrm{~km}$ and $660 \mathrm{~km}$ depth.

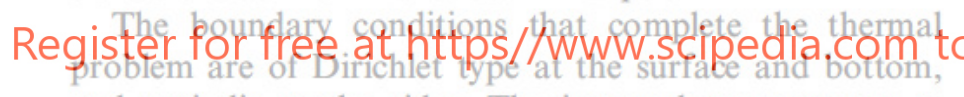

and periodic at the sides. The imposed temperatures at the surface and bottom are $T_{\text {surf }} 273 \mathrm{~K}$ and $T_{\text {bot }}$ $2053 \mathrm{~K}$, respectively. The sides are set as periodic to be consistent with the mechanical problem.

\section{Numerical approximation}

This section is devoted to describe the numerical methodologies used to solve the coupled transient nonlinear Eqs. (1) and (2). Note that, besides the standard difficulties associated with this type of problems, the numerical simulation of plate subduction requires describing and tracking the evolution of the different plates modelled as a multiphase system. In contrast to the common markers and cells method, here we use the eXtended Finite Element Method (X-FEM) instead. This approach adds two new ingredients to the standard finite element recipe: a level set and a functional enrichment via partitioning of the unity method.

\subsection{Level sets and phase tracking}

The interface between two materials is tracked using a level set function $\phi$. The sign of $\phi$ describes the geometrical domains using the following convention: $\phi(\mathbf{x}, t)= \begin{cases}>0 & \text { for } \mathbf{x} \text { in domain } 1 \\ =0 & \text { for } \mathbf{x} \text { on the interface } \\ <0 & \text { for } \mathbf{x} \text { in domain } 2\end{cases}$

where $\mathbf{x}$ stands for a point in the simulation domain and $t$ is the time. The interface position is the set of points where the level set field vanishes (Fig. 3). Initially, $\phi$ is taken as a signed distance to the interface. Far enough from the interface, $\phi$ is truncated by maximum and minimum cutoff values. The resulting level set function describes the position of the interface independently of the computational mesh.

In the practical implementation, $\phi$ is described (interpolated) with the finite element mesh, and therefore the resolution of the approximated interface depends on the quality of this mesh. Thus, the level set represents interfaces which do not necessarily coincide with the element edges. The same mesh can be used throughout the entire simulation to describe the interface.

The level set $\phi$ is a material property and consequently it is transported by the velocity. Therefore, it is updated by solving the following pure advection equation (first order hyperbolic)

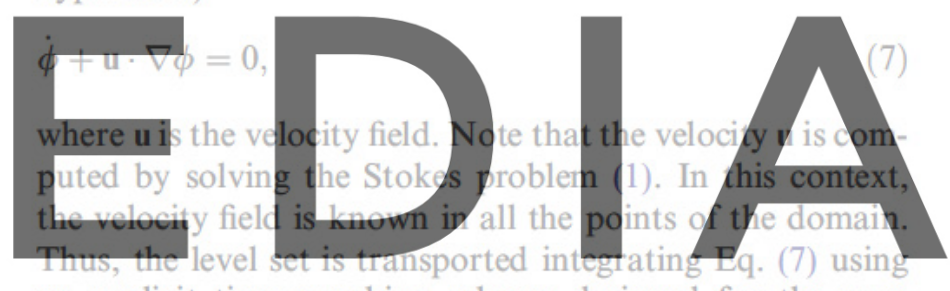

an explicit time-marching scheme designed for the pure


Galerkin method [15]. This method, described in Section 3.4, is straightforwardly implemented and computationally affordable. Note that in similar situations other authors use the Hamilton Jacobi equation to transport the interface. This is specially appropriate if the only data is the front velocity and if the front is sharp. In the present situation the velocity is known everywhere as a vector and it is possible to directly integrate the pure advection problem. In general, the time evolution of the level set function is such that it does not conserve the property of being a truncated distance to the interface (as set for the initial configura-
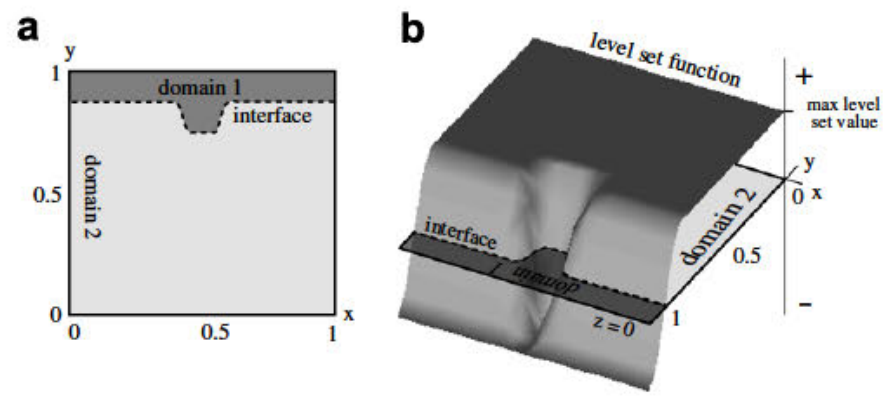

Fig. 3. (a) The two domains (materials) are associated with the sign of the level set function. The dotted line is the interface. (b) Surface represen tation of the level set function. 
tion). However, as demonstrated in the examples and in the test in Section 4.1, for the current application this method is sufficiently accurate and it does not require any post-process to reconstruct the distance shape.

The level set approach may describe changes in the shape (topology) of the phases. In practice, this allows the representation of detaching drops, merging bubbles, breaking sets, etc. This feature of the level set method is of great interest when used to model subduction processes. In some cases the slab may break off and separates from the surface plate sinking into the mantle. This topology change in the interface can be represented by the level set function.

\subsection{Space discretization}

The Stokes problem (1) is discretized using a mixed formulation, that is $\mathbf{u}$ and $p$ are interpolated differently. The stability of such a mixed formulation is guaranteed if the LBB condition is fulfilled. Here the well-known mini element [15] is used: this triangular element passes the LBB condition and has four nodes for the velocity (three at the vertices with linear shape functions and one at the cen-

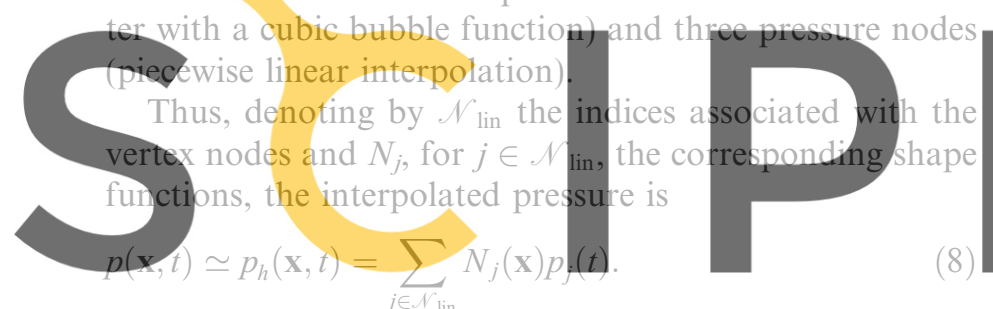

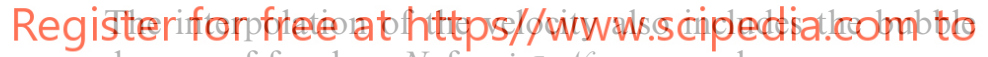
degrees of freedom $N_{j}$ for $j \in \mathcal{N}_{\text {bub }}$, namely

$\mathbf{u}(\mathbf{x}, t) \simeq \mathbf{u}_{h}(\mathbf{x}, t)=\sum_{j \in \mathcal{N}_{\text {lin }} \bigcup \mathcal{N}_{\text {bub }}} N_{j}(\mathbf{x}) \mathbf{u}_{j}(t)$.

The level set formulation is interpolated in terms of the linear degrees of freedom

$\phi(\mathbf{x}, t) \simeq \phi_{h}(\mathbf{x}, t)=\sum_{j \in \mathscr{N}_{\text {lin }}} N_{j}(\mathbf{x}) \phi_{j}(t)$.

In order to improve the ability of the interpolation to represent the gradient discontinuities across the interface, the interpolation of the velocity and pressure are enriched using a partition of the unity approach and a ridge function $R$, defined by

$R(\mathbf{x})=\sum_{j \in \mathcal{N}_{\mathrm{enr}}}\left|\phi_{j}\right| N_{j}(\mathbf{x})-\left|\sum_{j \in \mathcal{N}_{\mathrm{enr}}} \phi_{j} N_{j}(\mathbf{x})\right|$.

Note that $R$ is defined such that is only different from zero in the elements containing part of the interface [7]. The enrichment affects only the degrees of freedom corresponding to the vertex nodes of the elements in contact with the interface. The set on indices corresponding to such nodes is denoted as $\mathscr{N}_{\mathrm{enr}}$. Thus enriched interpolations of velocity and pressure are expressed as $\mathbf{u}_{h}(\mathbf{x}, t)=\sum_{j \in \mathcal{N}_{\text {lin }} \bigcup \mathscr{N}_{\text {bub }}} \mathbf{u}_{j}(t) N_{j}(\mathbf{x})+\sum_{j \in \mathcal{N}_{\text {enr }}} \mathbf{a}_{j}(t) M_{j}(\mathbf{x})$

and

$p_{h}(\mathbf{x}, t)=\sum_{j \in \mathscr{N}_{\text {lin }}} p_{j}(t) N_{j}(\mathbf{x})+\sum_{j \in \mathcal{N}_{\text {enr }}} b_{j}(t) M_{j}(\mathbf{x})$,

where $M_{j}(\mathbf{x}) \quad R(\mathbf{x}) N_{j}(\mathbf{x})$, and $\mathbf{a}_{j}$ and $b_{j}$ are the additional degrees of freedom for velocity and pressure, respectively.

A compact expression of the interpolation of velocity and pressure is used in the following:

$\mathbf{u}_{h}^{\mathrm{T}}(\mathbf{x}, t)=\mathbf{N}_{u} \mathbf{U}$

and

$p_{h}(\mathbf{x}, t)=\mathbf{N}_{p} \mathbf{P}$

where

$\mathbf{N}_{u}=\left[N_{1}, N_{2}, \ldots, N_{n_{u}}, M_{1}, \ldots, M_{n_{e}}\right]$,

$\mathbf{U}=\left[\mathbf{u}_{1}, \mathbf{u}_{2}, \ldots \mathbf{u}_{n_{u}}, \mathbf{a}_{1}, \ldots, \mathbf{a}_{n_{e}}\right]^{\mathrm{T}}$,

$\mathbf{N}_{p}=\left[N_{1}, N_{2}, \ldots, N_{n_{\text {lin }}}, M_{1}, \ldots, M_{n_{e}}\right]$,

$\mathbb{P}=\left[p_{1}, p_{2}, \ldots, p_{n_{\text {lin }}}, b_{1}, \ldots b_{n_{e}}\right]^{\mathrm{T}}$,

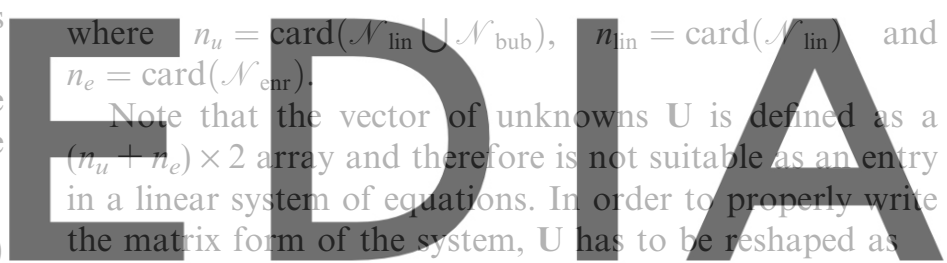

$\mathbf{U}^{\mathrm{rs}}=\left[u_{1}^{x}, u_{1}^{y}, u_{2}^{x}, u_{2}^{y}, \ldots, u_{n}^{x}, u_{n}^{y}, a_{1}^{x}, a_{1}^{y}, \ldots, a_{n}^{x}, a_{n}^{y}\right]^{\top}$ download the version without the watermark
which is a $2\left(n_{u}+n_{e}\right) \times 1$ vector. According to this reshape, a similar reordering has to be done in $\mathrm{N}_{u}$ in order to obtain an expression analogous to (14), namely

$\mathbf{N}_{u}^{\mathrm{rs}}=\left[\begin{array}{cccccccccccc}N_{1} & 0 & N_{2} & 0 & \ldots & N_{n_{u}} & 0 & M_{1} & 0 & \ldots & M_{n_{e}} & 0 \\ 0 & N_{1} & 0 & N_{2} & \ldots & 0 & N_{n_{u}} & 0 & M_{1} & \ldots & 0 & M_{n_{e}}\end{array}\right]$.

$\mathbf{N}_{u}^{\mathrm{rs}}$ is a $2 \times 2\left(n_{u}+n_{e}\right)$ matrix. It is worth noting that the number of enriched nodes, $n_{e}$ varies along time as the interface described by the level set is transported. Consequently, the size of $\mathbf{U}^{\mathrm{rs}}$ and $\mathbf{N}_{u}^{\mathrm{rs}}$ changes.

Problem (1) is discretized in space using the interpolation introduced above and the Galerkin formulation. The resulting system of algebraic equations reads

$\mathbf{K}_{u} \mathbf{U}^{\mathrm{rs}}+\mathbf{G}_{u}^{\mathrm{T}} \mathbf{P}=\mathbf{f}_{u}$,
$\mathbf{G}_{u} \mathbf{U}^{\mathrm{rs}}=\mathbf{0}$,

where the matrices $\mathbf{K}_{u}, \mathbf{G}_{u}$ and $\mathbf{f}_{u}$ are defined as

$$
\begin{aligned}
\mathbf{K}_{u} & =\int_{\Omega} \mathbf{B}^{\mathrm{T}} \eta \mathbf{B} \mathrm{d} V, \\
\mathbf{G}_{u} & =-\int_{\Omega} \mathbf{N}_{p}^{\mathrm{T}} \rho\left(\nabla \cdot \mathbf{N}_{u}^{\mathrm{rs}}\right) \mathrm{d} V, \\
\mathbf{f}_{u} & =\int_{\Omega}\left(\mathbf{N}_{u}^{\mathrm{rs}}\right)^{\mathrm{T}} \rho \mathbf{g} \mathrm{d} V .
\end{aligned}
$$


where the gravity vector $\mathbf{g}$ used in $\mathbf{f}_{u}$ is the column vector in the right hand side of Eq. (1a). The gradient matrix $\mathbf{B}$ is defined as

$$
\mathbf{B}=\left[\mathbf{B}_{1}, \mathbf{B}_{2}, \ldots, \mathbf{B}_{n_{u}}, \widetilde{\mathbf{B}}_{1}, \ldots, \widetilde{\mathbf{B}}_{n_{e}}\right],
$$

$\mathbf{B}_{i}=\left[\begin{array}{cc}\frac{\partial N_{i}}{\partial x_{1}} & 0 \\ 0 & \frac{\partial N_{i}}{\partial x_{2}} \\ \frac{\partial N_{i}}{\partial x_{2}} & \frac{\partial N_{i}}{\partial x_{1}}\end{array}\right] \quad$ and $\quad \widetilde{\mathbf{B}}_{i}=\left[\begin{array}{cc}\frac{\partial M_{i}}{\partial x_{1}} & 0 \\ 0 & \frac{\partial M_{i}}{\partial x_{2}} \\ \frac{\partial M_{i}}{\partial x_{2}} & \frac{\partial M_{i}}{\partial x_{1}}\end{array}\right]$.

Note that the spatial derivatives of $M_{i}$ contained in matrices $\widetilde{\mathbf{B}}_{i}$, for $i \quad 1, \ldots, n_{e}$ account for the enrichment and depend on the level set $\phi$. Therefore, the chain rule must be employed to evaluate those derivatives.

Eq. (16) are compacted in the following block matrix form $\lceil 151$

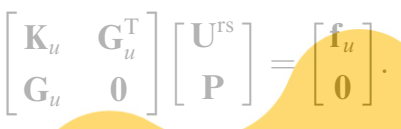

The discretization of the thermal problem (2) is performed using the linear interpolation, in a similar way as with pressure. The thermal conductivity is not expected to be discontinuous across the interface and therefore the interpolation

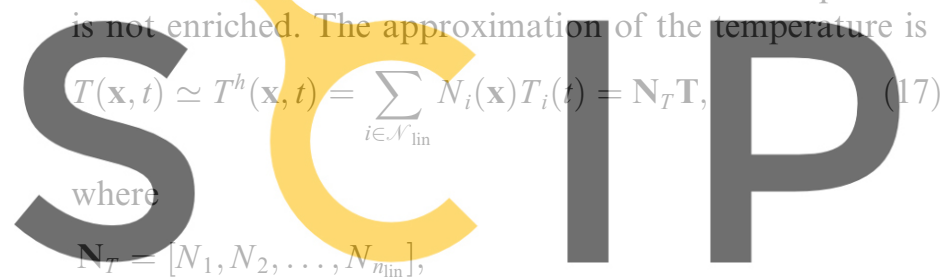
$\mathrm{T}=\left[T_{1}, T_{2}, \ldots, T_{n, \mathrm{in}}\right]$

Register for free at https//www.scipedia.com to And the system of ordinary differential equations resulting from the spatial discretization (17) reads

$\mathbf{M}_{T} \dot{\mathbf{T}}+\mathbf{G}_{T} \mathbf{T}=\mathbf{K}_{T} \mathbf{T}+\mathbf{f}_{T}$,

where

$$
\begin{aligned}
\mathbf{M}_{T} & =\int_{\Omega} \mathbf{N}_{T}^{\mathrm{T}} \rho C_{p} \mathbf{N}_{T} \mathrm{~d} V, \\
\mathbf{G}_{T} & =\int_{\Omega} \rho C_{p} \mathbf{N}_{T}^{\mathrm{T}} \mathbf{u}^{\mathrm{T}}\left(\nabla \mathbf{N}_{T}\right) \mathrm{d} V, \\
\mathbf{K}_{T} & =\int_{\Omega}\left(\nabla \mathbf{N}_{T}\right)^{\mathrm{T}} k \nabla \mathbf{N}_{T} \mathrm{~d} V, \\
\mathbf{f}_{T} & =\int_{\Omega} \mathbf{N}_{T}^{\mathrm{T}} q \mathrm{~d} V .
\end{aligned}
$$

The level set is discretized in space using a linear interpolation (same as with temperature)

$\phi(\mathbf{x}, t) \simeq \phi^{h}(\mathbf{x}, t)=\sum_{i \in \mathscr{N}_{\text {lin }}} N_{i}(\mathbf{x}) \phi_{i}(t)=\mathbf{N}_{T} \phi$,

where

$\phi=\left[\phi_{1}, \phi_{2}, \ldots, \phi_{n_{\operatorname{lin}}}\right]^{\mathrm{T}}$.

The transport equation of the level set (7) is discretized using (19) and yields
$\mathbf{M}_{\phi} \dot{\phi}-\mathbf{G}_{\phi} \phi=\mathbf{0}$,

where

$$
\begin{aligned}
& \mathbf{M}_{\phi}=\int_{\Omega} \mathbf{N}_{T}^{\mathrm{T}} \mathbf{N}_{T} \mathrm{~d} V, \\
& \mathbf{G}_{\phi}=-\int_{\Omega} \mathbf{N}_{T}^{\mathrm{T}} \mathbf{u}^{\mathrm{T}}\left(\nabla \mathbf{N}_{T}\right) \mathrm{d} V .
\end{aligned}
$$

\subsection{Integration quadrature for the multiphase elements}

In the elements cut by the level set, the functions to be integrated are discontinuous because the material properties are different at different sides of the level set. The standard Gauss quadratures used in the FE context are not well suited to integrate these discontinuous functions. There are two alternatives to obtain an accurate response in the multiphase elements: either to use a very large number of integration points or to decompose the element into simple one-phase subdomains for integration purposes. Here, we choose the second option because we are using triangular elements in 2D. Probably in more complex situations, especially en 3D, using a large number of integration points is

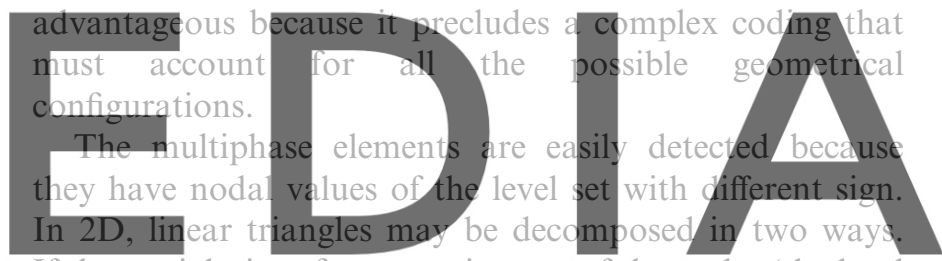

If the straight interface contains one of the nodes (the level set is zero in this node) the triangle is cut in two triangles. If

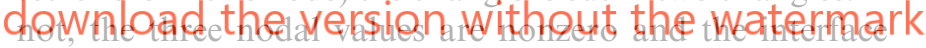

cuts two sides of the triangle. In this second case, the triangle is split in a new triangle plus a quadrilateral (which is cut into two triangles).

The implementation of this kind of quadrature requires using a numerical criterion to detect the intersection between the element and the interface. The results are sensitive to the tolerance used to detect if some point lies in the interface. This is because the ridge function takes a very small value when the level set is close to a node. In this case enriching the solution results in a ill-conditioned problem because the coefficient associated with the enriched part of the solution must compensate the tiny values of the ridge function. Here, the tolerance is set to a 10 percent of the element size.

\subsection{Time discretization}

The level set function tracking the interface is updated at each time step by the transport equation (7), which is rewritten as

$\dot{\phi}=-\mathbf{U} \cdot \nabla \phi$.

This equation is integrated upon time using a two-step third order Taylor Galerkin method (2S-TG3), namely 
$\tilde{\phi}^{n}=\phi^{n}+\frac{1}{3} \Delta t \dot{\phi}^{n}+\alpha \Delta t^{2} \ddot{\phi}^{n}$,

$\phi^{n+1}=\phi^{n}+\Delta t \dot{\phi}^{n}+\frac{1}{2} \Delta t^{2} \ddot{\tilde{\phi}}^{n}$.

The $\alpha$ parameter takes the value $1 / 9$ to reproduce the phase-speed characteristics of the single-step Taylor Galerkin scheme [15].

Taking into account the incompressibility equation (1b), the second time derivative of the level set function $\phi$ can be expressed as

$\ddot{\phi}=-\mathbf{u} \cdot \nabla(\dot{\phi})=\mathbf{u} \cdot \nabla(\mathbf{u} \cdot \nabla \phi)=\nabla \cdot((\mathbf{u} \cdot \mathbf{u}) \nabla \phi)$.

Therefore, the first step of the Taylor Galerkin algorithm is given by

$\tilde{\phi}^{n}=\phi^{n}+\frac{1}{3} \Delta t(-\mathbf{u} \cdot \nabla \phi)+\alpha \Delta t^{2} \nabla \cdot((\mathbf{u} \cdot \mathbf{u}) \nabla \phi)$.

Using the space discretization $\phi(19)$ of the level set function $\phi$, the first and second steps of the TG3 scheme are expressed in the following matrix forms

$\mathbf{M}_{\phi} \tilde{\phi}^{n}=\left[\mathbf{M}_{\phi}+\frac{1}{3} \Delta t \mathbf{G}_{\phi}+\alpha \Delta t^{2} \mathbf{K}_{\phi}\right] \phi^{n}$,

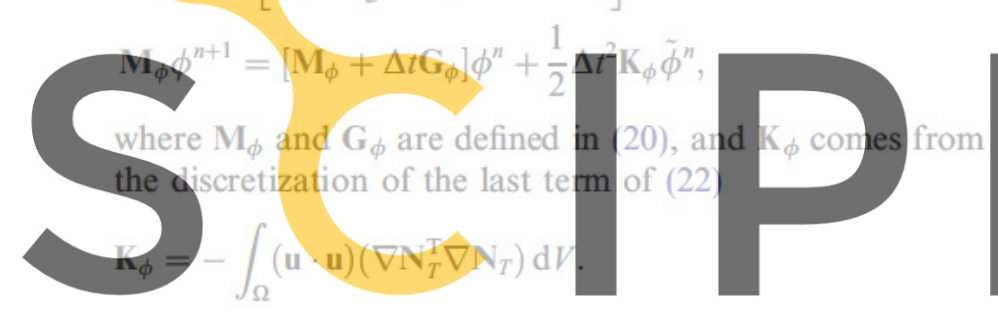

Note that $\mathbf{G}_{\phi}$ and $\mathbf{K}_{\phi}$ depend on the velocity field. In the

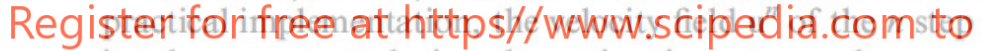
is taken constant during the entire time step and consequently the steady (quasi-static) Stokes problem is not solved for the intermediate step $\phi^{n}$.

To keep the solution in the stability domain of the twostep TG3 algorithm the time increment $\delta t$ is set such that the Courant number is lower than $3 / 4$.

The unsteady heat equation (2) is integrated upon time using a $n$-stage explicit Padé method. This multistage method is expressed in the following incremental form:
$T^{n+\beta_{1}}=T^{n}$,

$T^{n+\beta_{i}}=T^{n}+\beta_{i} \Delta t \dot{T}^{n+\beta_{i} 1}, \quad i=2, \ldots, n_{t g}+1$,

where $\beta_{1} \quad 0$, and $\beta_{i} \quad 1 /\left(n_{t g}+2-i\right)$.

From the discretization (18) of the heat equation (2), each step of the explicit Padé method can be written in the matrix form

$$
\begin{aligned}
& \mathbf{M}_{T} \dot{\mathbf{T}}^{n+\beta_{i 1}}=\left(\mathbf{G}_{T}+\mathbf{K}_{T}\right) \mathbf{T}^{n+\beta_{i 1}}+\mathbf{f}_{T}, \\
& \mathbf{T}^{n+\beta_{i}}=\mathbf{T}^{n}+\beta_{i} \Delta t \dot{\mathbf{T}}^{n+\beta_{i 1} 1} .
\end{aligned}
$$

This method updates the temperature from step $n$ to step $n+1$ computing the temperature in some intermediate points $n+\beta_{i}$. Same as with the TG3 integration algorithm, the $\mathbf{G}_{T}$ and $\mathbf{K}_{T}$ matrices depend on the velocity field $\mathbf{u}$ and in the present implementation a constant velocity is assumed during the time step. These matrices are assembled once in each time step, and then used in each stage of the Padé method.

\section{Validation test}

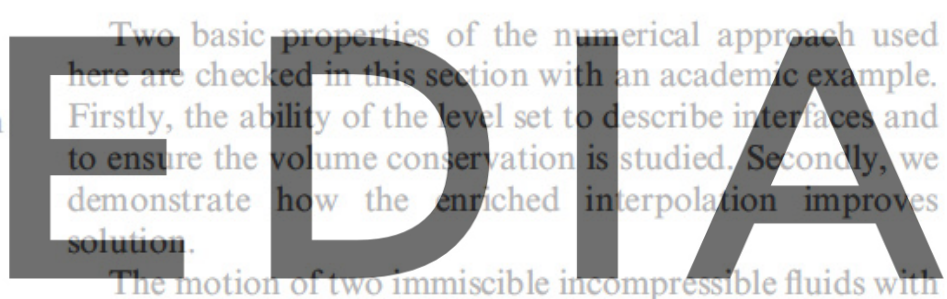

different densities and viscosities is simulated (the values

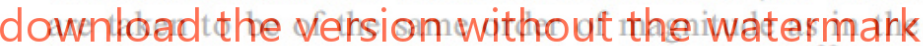
application example of the next section $\eta_{1} \quad 10^{22} \mathrm{~Pa} \mathrm{~s}$, $\rho_{1} \quad 3300 \mathrm{~kg} / \mathrm{m}^{3}$ and $\eta_{2} \quad 10^{20} \mathrm{~Pa} \mathrm{~s}, \rho_{2} \quad 3290 \mathrm{~kg} / \mathrm{m}^{3}$ ). At the initial configuration the denser fluid is in the top of a square box and it flows to the bottom. The initial state and several snapshots along the evolution are shown in Fig. 4. Note that in this example the thermal behaviour is not taken into account and the viscosity and density of each phase are assumed to be constant (linear mechanical model). This is because the goal of this example is only
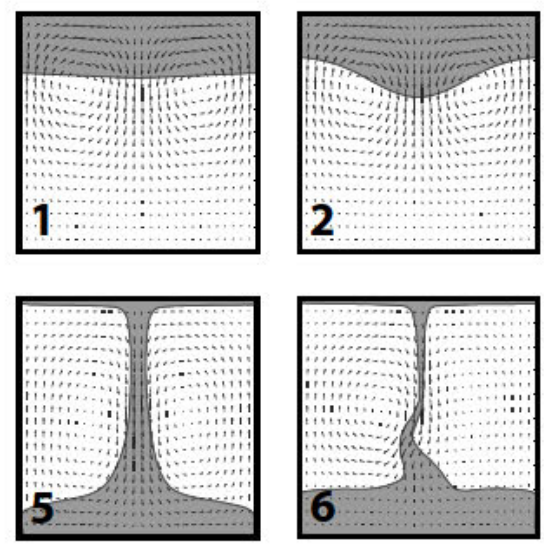
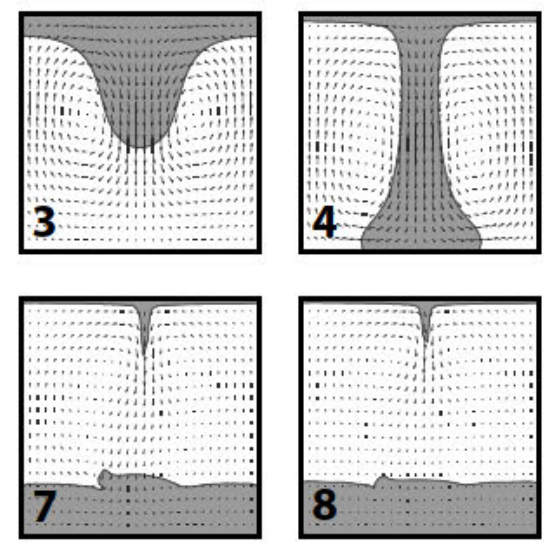

Fig. 4. Dense fluid falling in a square box. Evolution of the two phases. 
to test the description of the phase tracking via the level set approach (see Section 3.1), the accuracy of the numerical scheme used to transport the level set (solving Eq. (7)) and the influence of the enrichment in the numerical results.

\subsection{Checking volume conservation}

The volume of each phase (the denser and the lighter fluids) has to be conserved along the time stepping procedure. Therefore, volumetric changes in time are used to assess the accuracy of the numerical techniques.

The numerical test is performed with a series of uniformly refined non-structured meshes. The results are displayed in Table 1, which shows the maximum volume variation obtained with each mesh. As expected, the finer the mesh, the better description of the level set, and therefore, the lesser volume variation is observed. However, even with a coarser mesh (average edges length is 0.05 ) the error produced is less than $4 \%$.

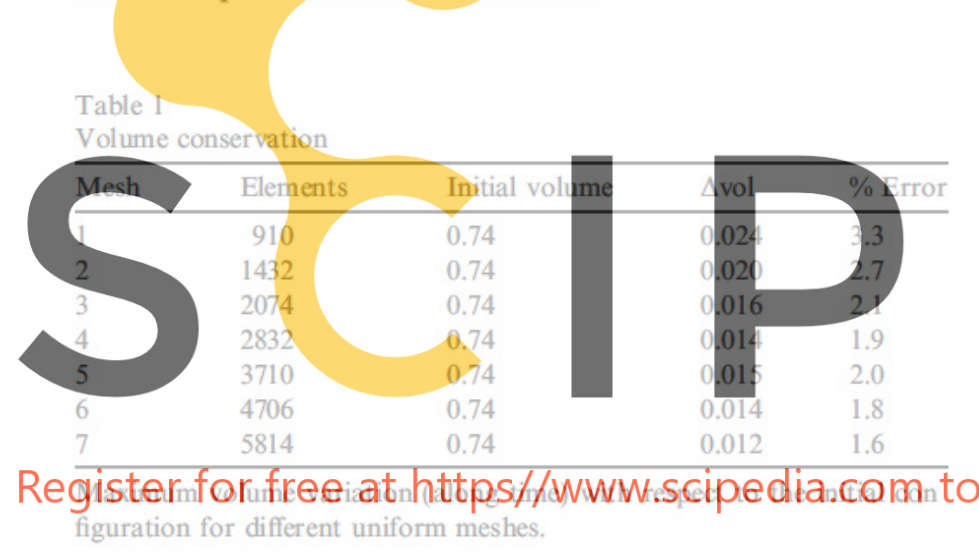

\subsection{Influence of the enrichment}

The influence of using the enrichment presented in Eqs. (12) and (13) is analyzed here. The mesh with 2074 elements (Fig. 5d) is taken as a reference for a coarser mesh of 508 elements (Fig. 5e). The solutions of the coarser mesh with and without enrichment are compared by computing the errors with respect to the more accurate solution of the finer reference mesh.

The solution is analyzed at a given time that corresponds to the configuration shown in Fig. 5c. The error is computed as the difference of the velocity solutions at a fine cloud of sample points. The global figures displayed in Table 2 demonstrate that the error is reduced using the enrichment both in its average and its maximum value by a factor of approximately 2 .

The enriched solution shows smaller errors (approx. one half) when compared with the non-enriched solution (Fig. 5a and b, respectively). The histogram of Fig. 5, shows that the lower errors for the enriched solution occur in a much larger number of points. In addition, the larger error (up to 3.5\%) are absent for the enriched solution.

This example demonstrates that, even in this simple problem, the enrichment is needed to properly capture
not only the discontinuities in the derivatives of the velocity
but also the values of the felocity itself.
Table 2
Error reduction by nrichment Enriched Not enriched dowiflorad the version'without the watermark
Average rel. error

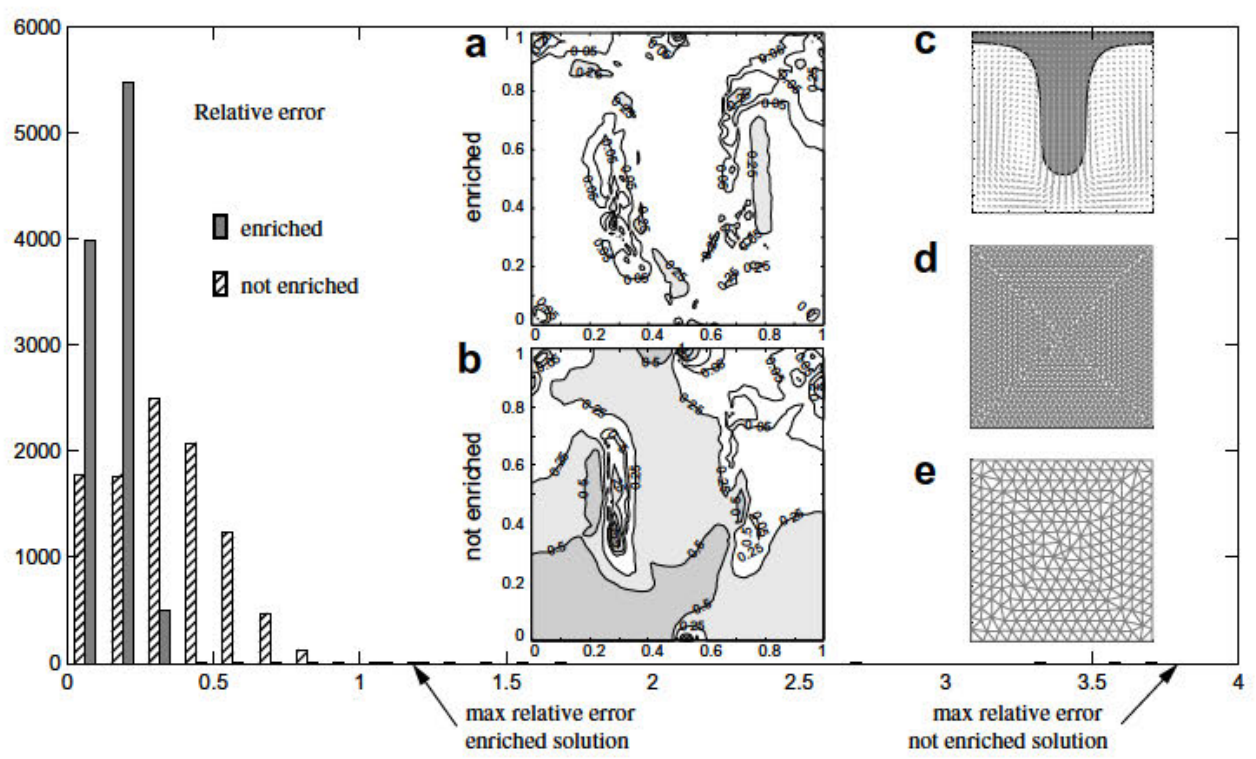

Fig. 5. Effect of the enrichment in the discretization error. Histogram of relative errors: number of occurrences of every error value at the sample points (left). Space distribution of relative errors for the enriched solution (a) and the not enriched solution (b). Configuration at the time analyzed (c). Finer mesh used in the reference solution (d) and coarser computational mesh (e). 


\section{Application example}

The convergence of two tectonic plates is simulated to model the subduction process, where one moving plate bends and sinks below the other plate. The relative velocity of convergence between tectonic plates is called convergence rate and does not refer to the approximation properties of any particular numerical scheme. The goal of the simulation is to find a relationship between the convergence rate and the geometry of the subducted oceanic plate $(s l a b)$. The geometry of the slab is parameterized by the slab dip (angle with respect to the horizontal) and the curvature at the discontinuity occurring at the depth of $660 \mathrm{~km}$.

Observations of real subduction zones reveal that the slab dip increases gradually from the surface to a depth of $80150 \mathrm{~km}$. Below this depth, it remains almost constant down to the limit between the upper and the lower mantle at $660 \mathrm{~km}$ depth, where the slab may deflect [16].

The problem statement is illustrated in Fig. 2a. The simulation is performed to reproduce approximately $1360 \mathrm{~km}$ of plate convergence, that is, one plate moves $1360 \mathrm{~km}$ against the other. The velocity of the plates at the surface


ual or sudden.

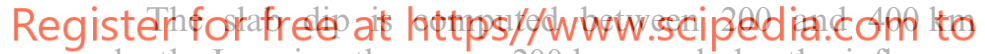
depth. Ignoring the upper $200 \mathrm{~km}$ precludes the influence from the shallow dip, possibly biased by the surface boundary conditions. The lower $400 \mathrm{~km}$ limit is above the curved part of the slab caused by the deflection at $660 \mathrm{~km}$.

The slab dip is computed by a linear least squares fitting of the position of the interface between the subducted
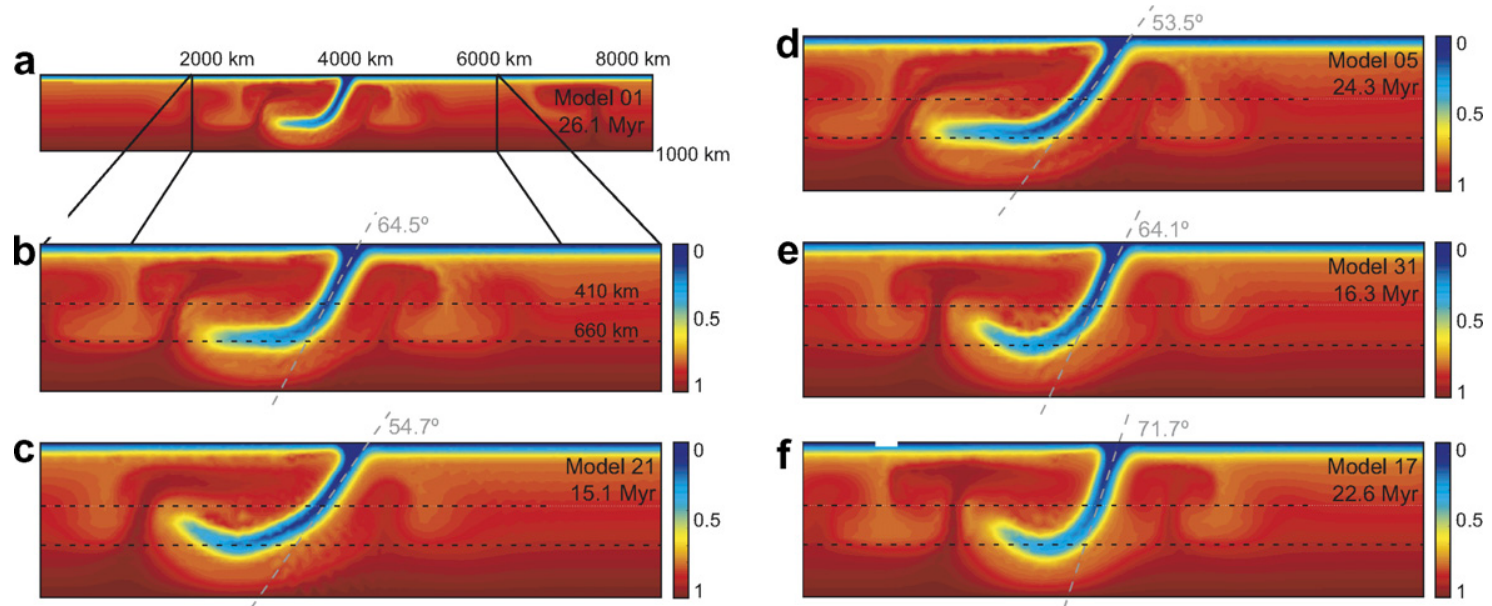

Fig. 6. Final temperature distributions (dimensionless) resulting in five different simulations. Complete computational domain (a) and zoom into the zone of interest (b) (f). The slab dip is indicated for each simulation. 
gives a correlation parameter of $R \quad-0.88$. If the linear regression is applied to the cases where the slab reaches the $660 \mathrm{~km}$ discontinuity (a grand total of 209 measures) the correlation is even better: $R \quad-0.93$.

An analysis of the relations between plate curvature at $660 \mathrm{~km}$ depth and other known parameters showed no evident correlation. At the end of every simulation, the slab remains buoyant at the $660 \mathrm{~km}$ discontinuity. Moreover, no systematic relationship is found between the final slab geometry and the type of velocity transition (sudden or gradual) or the acceleration of the slab (positive or negative).

Fig. 8 shows six velocity-dip paths, three corresponding to sudden velocity transitions and three to gradual transitions. It is observed that the average dip rate is $2 \% \mathrm{Myr}$ for shallowing slabs and $2.7^{\circ} / \mathrm{Myr}$ for steepening slabs.

The velocity-dip path for the model with a constant convergence rate of $10 \mathrm{~cm} / \mathrm{yr}$ (see Fig. 9) shows a clear increment in slab dip, until the plate is deflected at $660 \mathrm{~km}$ depth. Similar behaviour was found in other examples.

Geophysical interpretation. According to the simulations, the slab dip does not depend on the length of the subducted lithosphere, nor on the thermal state of the slab


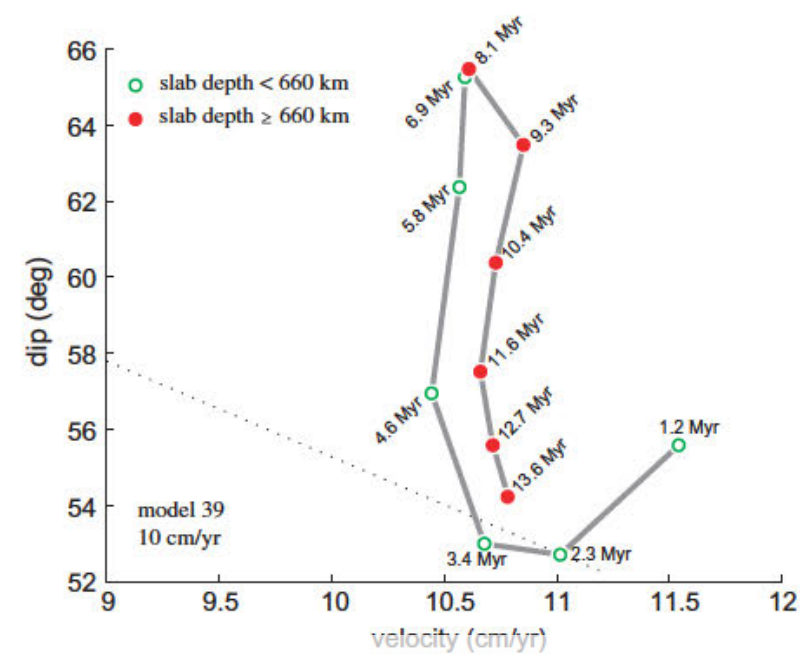

Fig. 9. Velocity dip path for a constant velocity model $(10 \mathrm{~cm} / \mathrm{yr})$.

its temperature to the surrounding mantle). A clear dependence is found between the slab dip and the convergence rate (velocity). The smoother velocity-dip paths correspond to the gradual velocity transition models and corroborate
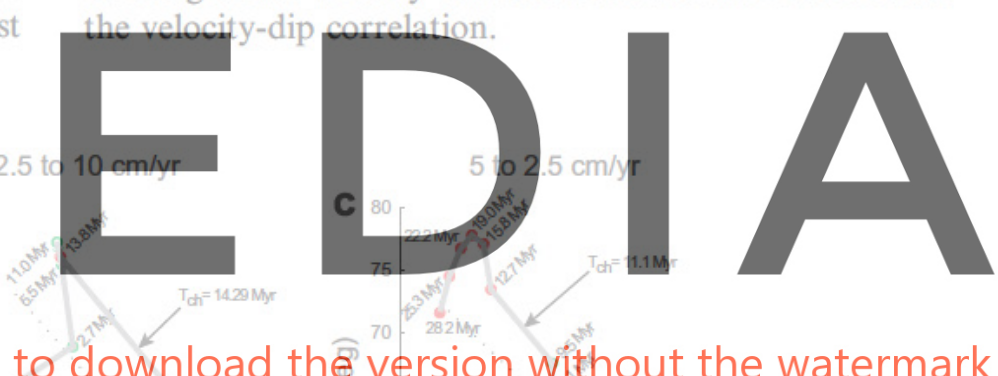

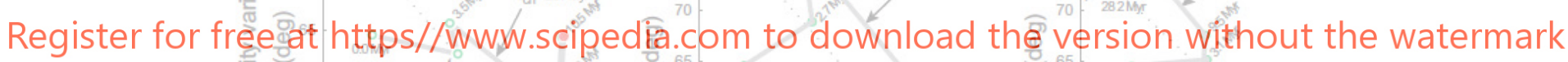
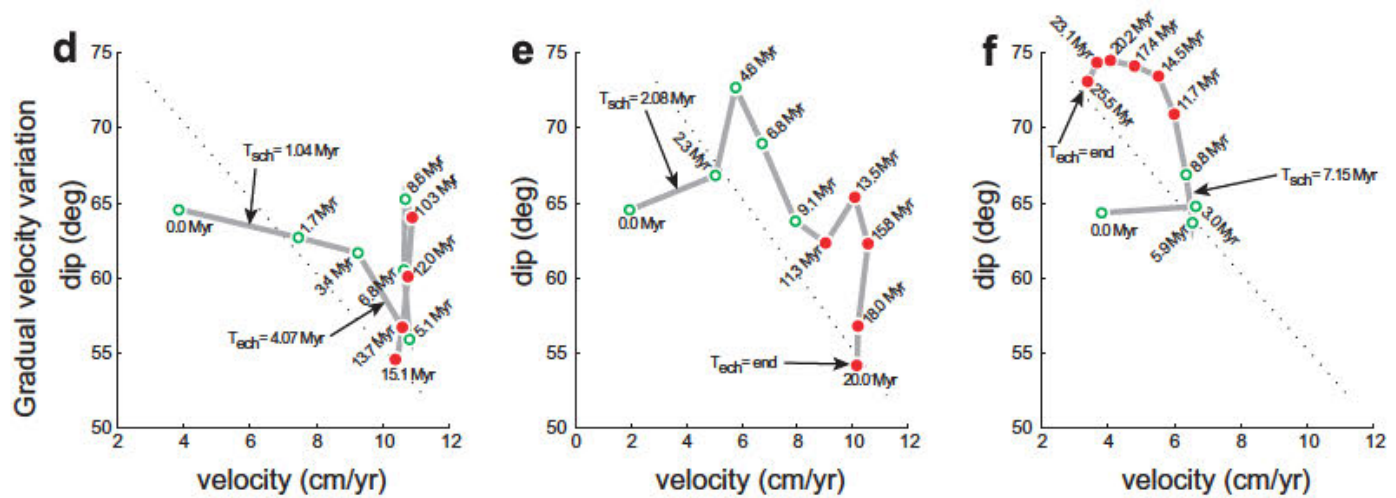

- slab depth $<660 \mathrm{~km}$

$T_{\text {ch }}$ time of sudden velocity variation

$T_{\text {sch }}$ start time of gradual velocity variation

$T_{\text {ech }}$ end time of gradual velocity variation

Fig. 8. Velocity dip paths for six shallowing and steepening models. Black arrows indicate the times where the velocity changes. Gradual transition models have smoother paths than sudden transition models with same initial and final velocity. 
We interpreted that two forces are in competition to control the dip: the viscous force of the mantle resisting the slab penetration, which depends on the plate velocity, and the mass excess caused by the lateral density contrast, depending on the temperature contrast between the slab and the mantle (the slab-pull force). If, as it is found in the present simulations, the velocity correlates with the slab dip, the mechanical viscous forces have a greater influence in the slab geometry than the gravitational forces. The effect of the thermal state is in this case of second order.

\section{Concluding remarks}

A numerical model of the tectonic plate subduction is presented.

The physical model accounts for the complexity of this natural system and its main features are summarized in the following points:

- the pseudo-static mechanical problem (inertia terms are neglected and therefore no time derivatives appear) is governed by the Stokes equation, being a two-phase fluid with a sharp viscosity contrast,

- highly-nonlinear rheology,

- two-way mechanical thermal coupling, being the thermal problem advection dominated and with a heat production term associated with shear effects, based on strain rate,

- the physical properties depend on both temperature and pressure.

The resulting model is numerically solved using the $\mathrm{X}-\mathrm{FEM}$ approach for the space discretization and explicit time-marching schemes which handle properly the advective character of the problem.

The results of the numerical tests allow assessing the relation between the plate convergence velocity and the subducted plate dip. These observations are relevant for the geophysical interpretation of the model. A clear correlation between these subduction parameters is found and it is concluded that the mechanical viscous forces have a larger influence on the dip than the thermal forces. These con- clusions may be extended to assess the origin of the driving forces of the plate motion in the subduction zones.

\section{References}

[1] B. Schott, H. Schmeling, Delamination and detachment of a lithospheric root, Tectonophysics 296 (1998) 225247.

[2] L. Moresi, F. Dufour, H. B. Muhlhaus, A lagrangian integration point finite element method for large deformation modeling of viscoelastic geomaterials, J. Comput. Phys. 184 (2003) 476497.

[3] T.V. Gerya, D.A. Yuen, Characteristics based marker in cell method with conservative finite differences schemes for modeling geological flows with strongly variable transport properties, Phys. Earth Planet. Inter. 140 (4) (2003) 293318.

[4] R. Pysklywec, M. Ishii, Time dependent subduction dynamics driven by the instability of stagnant slabs in the transition zone, Phys. Earth Planet. Inter. 149 (2005) 115132

[5] V.C. Manea, M. Manea, V. Kostoglodov, G. Sewell, Intraslab seismicity and thermal stress in the subducted cocos plate beneath Central Mexico, Tectonophysics 420 (3 4) (2006) 389408.

[6] T. Belytschko, T. Black, Elastic crack growth in finite elements with minimal remeshing, Int. J. Numer. Methods Engrg. 45 (5) (1999) 601 620.

[7] N. Moes, M. Cloirec, P. Cartaud, J.F. Remacle, A computational approach to handle complex microstructure geometries, Comput. Methods Appl. Mech. Engrg. 192 (2003) 31633177.

[8] J. Chessa, T. Belytschko, An extended finite element method for two phase fluids, Trans. ASME (2003) 1017.

[9] T.V. Gerya, D.A. Yuen, W.V. Maresch, Thermomechanical model ling of slab detachment, Earth Planet. Sci. Lett. 226 (2004) 101116.

[10] V.C. Manea, M. Manea, V. Kostoglodov, G. Sewell, Thermo mechanical model of the mantle wedge in Central Mexican subduc tion zone and a blob tracing approach for the magma transport, Phys. Earth Planet. Inter. 149 (1 2) (2005) 165186

[11] G. Schubert, D.L. Turcotte, P. Olson, Mantle Convection in Earth and Planets, Cambridge University Press, UK, 2001.

[12] A.M. Hofmeister, Mantle values of thermal conductivity and the geotherm from phonon lifetimes, Science 283 (1999) 19691706.

[13] C. Clauser, E. Huenges, Thermal conductivity of rocks and minerals, in: T. Ahren (Ed.), Rock Physics and Phase Relations, third ed., AGU Reference Shelf, AGU, Washington DC, 1995, part 3, pp. 105126.

[14] G. Ranalli, Rheology of the Earth, second ed., Chapman and Hall, 2 6 Boundary Row, London, 1995.

[15] J. Donea, A. Huerta, Finite Element Methods for Flow Problems, Wiley, Chichester, West Sussex PO19 8SQ, England, 2002.

[16] S. Lallemand, A. Heuret, On the relationships between slab dip, back arc stress, upper plate absolute motion, and crustal nature in subduction zones, Geochem. Geophys. Geosyst. 6 (9). doi:10.1029/ 2005 GC000917. 